\title{
Evaluation of Unilateral Vocal Fold Immobility
}

\author{
Robbi A. Kupfer · Tanya K. Meyer
}

Published online: 21 February 2014

(c) Springer Science+Business Media New York 2014

\begin{abstract}
Unilateral vocal fold immobility (UFVI) is a highly variable clinical entity that may be neurogenic or mechanical in origin. Patients with UVFI have varying degrees of voice, swallowing, and airway disturbance. The patient should be carefully evaluated, including thorough history, head, neck, and neurologic examination, and laryngoscopy, to determine the etiology of UFVI and the current level of laryngeal function. If the etiology cannot be determined, cross sectional imaging of the skull base through the mediastinum is recommended to evaluate the underlying cause. Laryngeal electromyography is most useful for predicting poor functional recovery and may affect the decision to proceed with early definitive surgical management. Formal swallowing testing is indicated for patients with dysphagia to identify aspiration.
\end{abstract}

Keywords Unilateral vocal fold immobility · Vocal cord paralysis · Vocal fold paralysis - Laryngeal electromyography

\section{Introduction}

Located at the junction of the respiratory and digestive tracts, the larynx is a complex organ with essential functions in respiration, swallowing, phonation, and cough production. Normal laryngeal physiology depends upon

R. A. Kupfer $(\bowtie) \cdot$ T. K. Meyer Department of Otolaryngology-Head and Neck Surgery, University of Washington, 1959 NE Pacific Street, Suite BB1165, Box 356515, Seattle, WA 98195-6515, USA e-mail: rkupfer@uw.edu highly coordinated motor function, and disruption of this elegantly balanced system by vocal fold motion impairment may affect any of the vital laryngeal functions. This article will review the causes of unilateral vocal fold immobility (UFVI) and present an approach to investigation of patients with this diagnosis. Thorough evaluation of the patient is essential to establish the etiology of UVFI, to assess the extent of dysfunction, and to determine the appropriate management.

\section{Causes of Vocal Fold Immobility}

The causes of UVFI may be broadly divided into neurogenic or mechanical etiology (Tables 1,2). Evaluation and management differ substantially between these two categories, making it important to determine the cause of motion impairment and use accurate terminology. Vocal fold immobility describes motion impairment resulting from any cause, whereas vocal fold paralysis specifically implies vocal fold immobility because of neurologic injury. Although neurogenic dysfunction accounts for most unilateral motion impairment cases, the term vocal fold immobility should be used to describe cases of vocal fold motion impairment unless a neurogenic etiology is confirmed by either electromyography or a convincing clinical history of recurrent laryngeal nerve (RLN) or vagus nerve injury.

It is important to note that vocal fold paralysis does not indicate complete absence of neurologic activity; rather, the larynx has a propensity for re-innervation that is nonselective and typically does not result in meaningful vocal fold movement. Vocal fold paresis refers to hypomobility of the vocal fold that is neurogenic in nature, implying partial neurologic injury. 
Table 1 Causes of vocal fold immobility

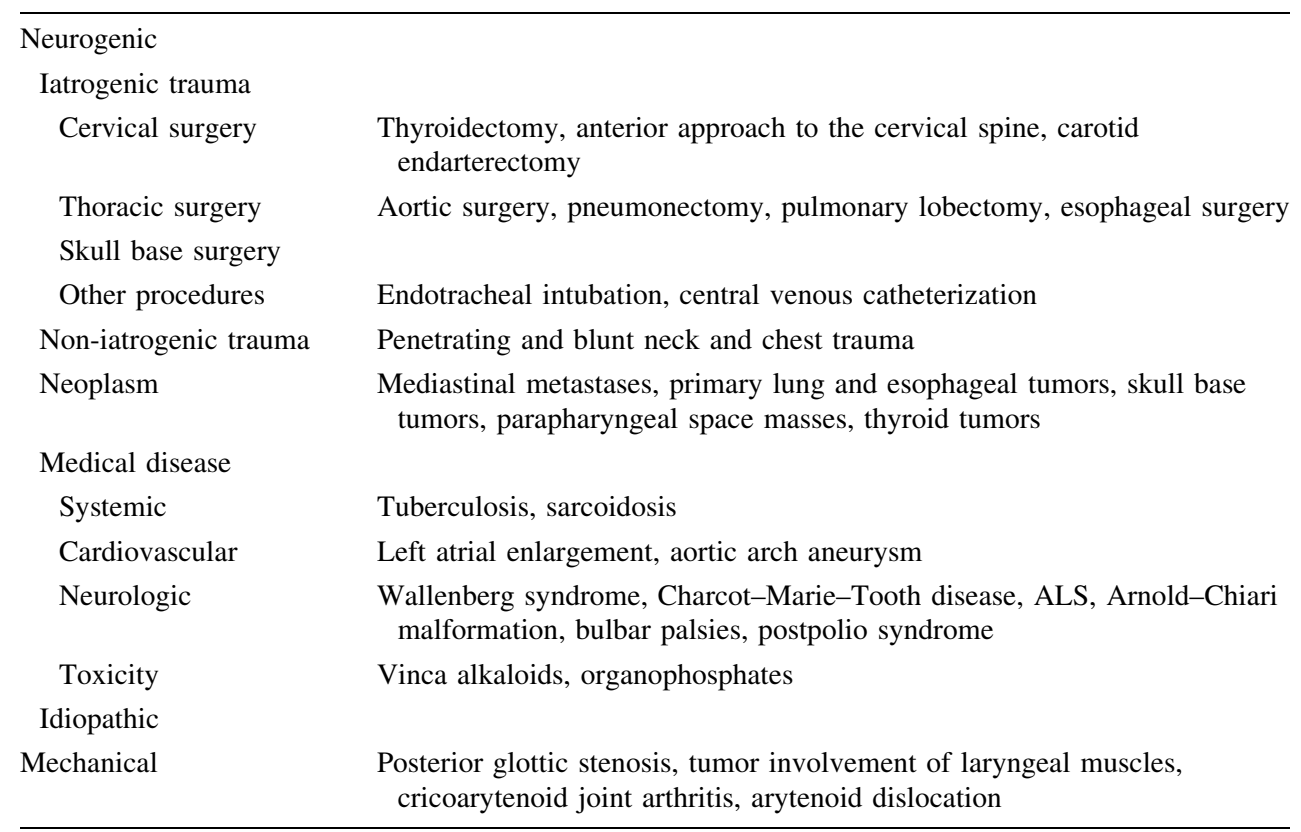

Table 2 Etiology of vocal fold paralysis

\begin{tabular}{lc}
\hline Etiology & Percentage of cases [1-5] \\
\hline Iatrogenic trauma & $18.8-51.5$ \\
Non-iatrogenic trauma & $1.2-11.2$ \\
Neoplasm & $13.5-40.5$ \\
Medical disease & $6-18.9$ \\
Idiopathic & $10.7-18.5$ \\
\hline
\end{tabular}

Injury to the RLN or vagus nerve may occur as a result of a wide range of etiology, including iatrogenic trauma, non-iatrogenic trauma, neoplasm, and systemic disease [15]. The lengthy course of the RLNs places them at high risk of injury, particularly the left nerve, which loops around the aorta. Consequently, the incidence of left UVFI accounts for $\sim 60 \%$ of UVFI cases $[3,4]$. Injury to the vagus nerve proximal to the branching point of the superior laryngeal nerve is known as a "high vagal" injury and, clinically, often results in a more severe presentation, particularly with greater degrees of dysphagia because of loss of sensation and motion impairment.

Surgical trauma may occur at any point along the nerves, and the procedures most likely to result in RLN or vagus nerve injury include brainstem, skull base, neck, and thoracic surgery. Historically, thyroid surgery was the procedure most commonly implicated in vocal fold paralysis, with reports of injury to the RLN occurring in $0.3-13.2 \%$ of cases [6]. Increasing use of the anterior approach for cervical spine surgery has resulted in an increase of vocal fold paralysis cases associated with cervical spine surgery, with reported incidence of 2-21.6\% of all anterior cervical spine surgery [6]. In contemporary studies of UVFI, thyroid surgery accounts for 12-26\% of surgical causes of UVFI, and cervical spine surgery is implicated in 15-32\% [4, 5]. Although the proportion of iatrogenic cases of UVFI caused by thyroid surgery seems to be decreasing, the vast majority $(80 \%)$ of surgicallyinduced bilateral vocal fold immobility can still be attributed to thyroid and parathyroid surgery [5].

Thoracic procedures that place the RLNs at risk of injury include aortic surgery, esophageal surgery, pulmonary lobectomy, pneumonectomy, mediastinoscopy, and thymectomy. Surgery involving the brainstem and skull base may result in vagus nerve paralysis and is often associated with other neurologic deficits. Other iatrogenic trauma includes endotracheal intubation, occurring in $0.1 \%$ of patients intubated for any reason, and central venous catheterization $[7,8]$.

Compression or involvement of the vagus nerve or RLN by a tumor may also occur at any location on the nerve from the skull base through the upper thoracic cavity. Although mediastinal metastases are the most common neoplastic cause of UVFI, vagal neuromas, parapharyngeal space masses, thyroid tumors, cervical metastases, esophageal tumors, thymomas, and primary lung tumors are also frequently implicated.

A variety of systemic processes and medical diseases may result in UVFI. Granulomatous disease, including sarcoidosis and tuberculosis, may cause injury to or compression of the RLN and vagus nerves. Cardiovascular disease, for example left atrial enlargement or aortic arch aneurysm may result in left RLN compression and vocal fold paralysis, known as cardiovocal or Ortner's syndrome. 
Neurotoxic medications, for example vinca alkaloids, and exposure to other neurotoxins have been implicated in both unilateral and bilateral vocal fold paralysis cases.

Vocal fold paralysis and paresis may also occur in association with several central or peripheral neurologic disorders. Patients with these will often have other neurologic signs and symptoms that help establish the diagnosis. Wallenberg syndrome, resulting from lesions or strokes of the lateral medulla, leads to ipsilateral vagal paralysis, including loss of pharyngeal sensation. Patients with Wallenberg syndrome also have ipsilateral Horner's syndrome and loss of pain and temperature sensation of the contralateral trunk and extremities [9]. Arnold Chiari malformation may result in compression of the vagus nerves at the skull base, typically presenting as bilateral vocal fold paralysis in the pediatric population. CharcotMarie-Tooth disease is a hereditary peripheral nerve palsy that infrequently results in laryngeal neuropathy, which most often presents as progressive bilateral vocal fold paresis $[10,11]$. Vocal fold paralysis may also be among the neurologic deficits caused by amyotrophic lateral sclerosis (ALS), postpolio syndrome, and bulbar palsies.

Although comprehensive evaluation will often identify the cause of UVFI, the etiology remains elusive in $10-20 \%$ of cases. These idiopathic cases are presumed to be secondary to viral neuropathy, suggested by positive serology and antecedent viral illnesses in a small proportion of cases.

Vocal fold immobility from mechanical causes may result from processes involving the cricoarytenoid joint or intrinsic laryngeal muscles. Prolonged intubation and other laryngeal trauma may result in posterior glottic stenosis or fibrosis of the cricoarytenoid joint. UVFI may also occur as a result of rheumatoid arthritis involving the cricoarytenoid joint, fibrosis secondary to radiation therapy, and invasion of the intrinsic laryngeal muscles by tumors. Dislocation of the arytenoid cartilage has been described, with intubation trauma implicated in most of these cases [12]. The topic of arytenoid dislocation and subluxation off the cricoarytenoid joint is regarded as controversial by many authors. One recent well executed study demonstrated that endotracheal intubation invoked trauma cannot reliably mobilize or disrupt this joint [13].

\section{History}

Evaluation of the patient with UVFI should include an assessment of the patient's symptoms and a search for the etiology. Symptoms of UFVI are the result of glottic insufficiency, and typically manifest as a weak, breathy, and hoarse voice. The severity of voice complaints ranges from mild vocal fatigue to severe dysphonia, typically correlating with the degree of glottic insufficiency present [14]. Increased vocal effort, vocal fatigue, odynophonia, and difficulty being heard over background noise or on the telephone are common complaints. Patient-reported outcomes from measurement of voice-related quality of life (V-RQOL) are useful for assessing the effect of dysphonia on the patient and for comparing scores to determine response to treatment. Several instruments are available, including the V-RQOL measure [15] and the voice handicap index (VHI) [16].

Patients with UVFI also frequently complain of dysphagia, particularly aspiration of liquids. A persistent glottic gap, associated sensory deficits, pharyngeal weakness, and delayed relaxation of the cricopharyngeus muscle may all contribute to dysphagia in UFVI cases. A thorough swallowing history should include a discussion of their current diet, difficulty swallowing liquids or solid foods, compensatory strategies they have developed, weight loss, and history of pneumonia.

Dysphagia associated with UVFI may have a wide range of severity, affected by level of neurologic injury (e.g. central nervous system, proximal vagus nerve, or RLN), pulmonary function, medical comorbidities, and overall vigor of the patient. Although isolated RLN injury rarely results in severe dysphagia, frank aspiration is common in patients with laryngeal sensory deficits and pharyngeal weakness from high vagal injury or who have other associated cranial neuropathies from a CNS process. The same level of aspiration that is well tolerated by patients with a strong cough may be debilitating for those patients with severely diminished cough mechanism. Similarly, underlying pulmonary pathology, for example asthma or chronic obstructive pulmonary disease (COPD), inhibits a patient's ability to safely tolerate mild aspiration.

Dyspnea is commonly experienced by patients with UVFI, and may result from two very different mechanisms. Most often, patients have a sensation of breathlessness during speech that results from escape of air through the glottic gap during phonation. Cough mechanism and thoracic stabilization during the Valsalva maneuver are also affected. Less frequently, patients may experience dyspnea as a result of partial glottic obstruction, as a result of inability to fully abduct the immobile vocal fold. In the absence of contralateral vocal fold motion impairment, most patients will be asymptomatic from the small decrease in glottic opening, except for those at the extremes of fitness. For patients with dyspnea, differentiating these two causes of their symptoms is important for determining appropriate surgical management, as the treatment for one would only exacerbate the other.

In determining the etiology of vocal fold immobility, past medical and surgical histories often reveal a likely source of injury. A history of diseases or procedures 
affecting the brain, skull base, neck, and chest, and history of antecedent intubation should be investigated. Systemic medical disease, for example sarcoidosis and rheumatoid arthritis, should be identified. Clinicians should perform a thorough neurologic review of systems to uncover any underlying neurologic disorders. A history of tobacco use is important to determine risk of pulmonary malignancy and guide decisions for further evaluation with imaging.

\section{Physical Examination}

\section{Perceptual Analysis of the Voice}

Evaluation of the patient with suspected UVFI should include assessment of voice as a crucial element of the physical examination. Some patterns of voice disturbances are characteristic of UVFI. A breathy quality of the voice will result from a persistent glottic gap that allows inappropriate air escape during phonation. Inability to loudly project the voice is common among patients with flaccidity of the vocal fold from paralysis. Diplophonia may occur when the immobile vocal fold is at a different tension from that of the contralateral side, resulting in each vocal fold vibrating at a different frequency. This may be most noticeable when the patient tries to yell, as increased subglottic pressure causes the flaccid vocal fold to luff away from midline.

Compensatory mechanisms may also result in abnormal voice patterns that are characteristic of vocal fold immobility. Many patients will have a strained quality of their voice from extralaryngeal muscle hyperfunction to compensate for glottic insufficiency. Elongation and tension of the vocal folds via cricothyroid muscle activation is another compensatory phenomenon that reduces the glottic gap and resultant breathiness but results in an abnormally high-pitched voice, known as compensatory or paralytic falsetto [17]. The presence of this phenomenon suggests an intact superior laryngeal nerve. The clinician should also listen for disturbances in articulation, fluency, and resonance, which would raise suspicion for a proximal neurologic injury or underlying neurologic disorder.

Some significant speech disturbances may only be identified by asking the patient to perform specific tasks. Maximum phonation time (MPT) is a simple bedside test that measures the duration a patient can sustain the vowel/i/ on a single breath. Although highly dependent upon patient effort, this test enables quantification of glottic insufficiency and is particularly useful for assessing outcomes of treatment. An MPT of $>21 \mathrm{~s}$ is considered normal. Another test of glottic insufficiency is the $\mathrm{s} / \mathrm{z}$ ratio, which compares the maximum time a patient can sustain an unvoiced $/ \mathrm{s} /$ to a voiced /z/. Patients with glottic insufficiency will have decreased maximum phonation time on a /z/ whereas the /s/ time should remain near normal, resulting in an increased $\mathrm{s} / \mathrm{z}$ ratio. Articulation, muscle control, and coordination may be tested by rapid repetition of the syllable /pa/ for lip function, /ta/ for tongue tip function, and /ga/ for posterior tongue function.

\section{Head and Neck Examination}

A thorough and systematic neurologic examination of the head and neck should be performed for all patients with UVFI [18]. All cranial nerves should be tested, with particular attention to the other branches of the vagus nerve and the hypoglossal and accessory nerves, which travel with the vagus nerve through the jugular foramen. Palatal asymmetry is assessed during elevation with phonation. Tongue deviation indicates hypoglossal weakness, although a more subtle tongue weakness may be discovered by having the patient push the tongue against the cheek while it is palpated by the examiner externally [19]. The tongue should be examined at rest for signs of fasciculations. Ocular examination should be performed to check for Horner's syndrome. The neck should also be carefully examined for masses, lymphadenopathy, and surgical scars.

\section{Laryngeal Examination}

Laryngeal examination is best accomplished with transnasal flexible laryngoscopy, because this enables observation of pharyngeal and laryngeal motor function. Although rigid laryngoscopy enables excellent visualization of the endolarynx, the pharynx and soft palate are not examined in as much detail with this technique and tongue traction required may alter laryngeal posture and lead to an inaccurate assessment of laryngeal function.

The range of laryngoscopic findings in vocal fold immobility is quite variable (Fig. 1). This is not surprising, given the array of different etiology of vocal fold immobility and the range of innervation status among cases of vocal fold paralysis. Furthermore, there is little consensus among laryngologists' interpretation of laryngoscopic findings in vocal fold motion impairment [20]. Nevertheless, a thorough laryngoscopic examination is critical for determining an individual patient's laryngeal function and most appropriate management.

The larynx should be examined during a variety of tasks. Instructing the patient to alternate between a sustained vowel /i/ and sniffing through the nose (/i/-sniff maneuver) will result in maximum adduction and abduction and reveal asymmetric vocal fold motion. UVFI is readily detectable by laryngoscopy for most patients, although cricothyroid, interarytenoid, and extralaryngeal muscle function may result in slight movement that leads to diagnostic challenges. Intact contralateral innervation of the interarytenoid 
Fig. 1 Variable laryngoscopic findings for two individuals with left vocal fold paralysis. Laryngoscopy for patient a reveals anterior displacement of the arytenoid, flaccidity of the true vocal fold, and salivary pooling in the left piriform sinus. In contrast, patient $\mathbf{b}$ has a fairly upright arytenoid with atrophic vocal fold with only minimal flaccidity

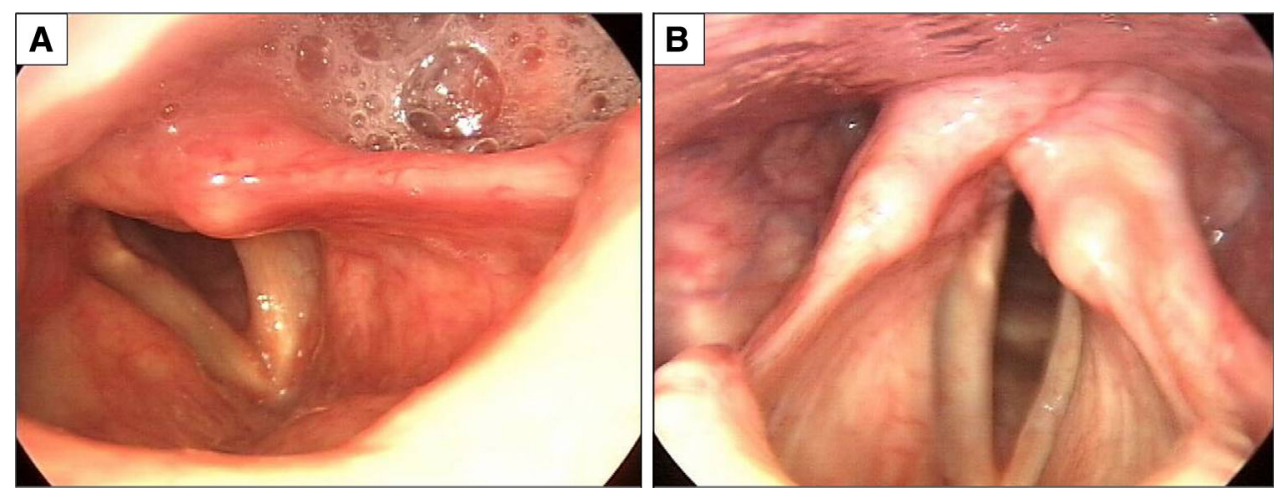

muscle may cause subtle adduction of a paralyzed vocal fold during phonation, resulting in apparent abduction. Asking the patient to perform a single sniff while the vocal folds are at rest is the most accurate test for isolation of vocal fold paralysis in the setting of interarytenoid movement; a paralyzed vocal fold from RLN injury cannot actively abduct from the resting position. Conversely, a paralyzed vocal fold may paradoxically adduct during inspiration, either from passive medialization of a denervated flaccid vocal fold by Bernoulli effect or from inappropriate adduction caused by synkinesis.

Hyperfunction of extralaryngeal muscles is frequently used by patients with UVFI to compensate for glottic insufficiency. The resulting anteroposterior compression of the glottis and hyperadduction of the ventricular folds may obscure the view of the glottis during laryngoscopy and may be the most prominent sign of glottic insufficiency [21]. "Unloading" the voice with strategies to reduce compensatory hyperfunction, for example sighing and humming, can be performed during laryngoscopy to improve visualization of the true vocal folds.

The position of the arytenoid cartilage is often altered in cases of vocal fold immobility. The arytenoid cartilage is stabilized on the cricoid by the intrinsic laryngeal muscles, particularly the posterior cricoarytenoid muscle. Paralysis of these muscles may lead to anteromedial prolapse of the arytenoid apex and caudal displacement of the vocal process. The arytenoid may also demonstrate the "jostle sign" from lateral displacement by contact with the arytenoid on the intact side [22]. This is most readily detected by observing lateral motion of the muscular process of the paralyzed arytenoid under the mucosa of the piriform sinus. Presence of this passive arytenoid movement is highly suggestive of a mobile arytenoid and may be useful in cases in which cricoarytenoid joint fixation is suspected.

Glottic configuration and the position of the true vocal folds should be assessed in detail, in both the medial-lateral and rostral-caudal planes. Although, historically, it was believed that the position of the paralyzed vocal fold could be used to determine the etiology of vocal fold immobility and the site of neurologic injury, several studies have since shown no correlation between site of injury and vocal fold position [23-25]. However, systematic examination of vocal fold position is essential for surgical planning. The most consistent glottic findings in UVFI are a shortened and bowed vocal fold [23]. Anterior rotation of the arytenoid results in caudal displacement of the muscular process and a vertical height mismatch of the paired vocal folds. It is important to recognize height mismatch before surgical intervention, because medialization alone may fail to achieve satisfactory voicing. The glottic closure pattern should be assessed for contact both anteriorly, between the vibrating edges of the membranous vocal folds, and posteriorly at the vocal processes. Atrophy and bowing of both vocal folds should be noted, as significant bowing of the contralateral mobile vocal fold may prevent adequate glottic closure even after the immobile side has been appropriately medialized.

In addition to the larynx, other structures of the upper aerodigestive tract are examined during flexible laryngoscopy. Examination of the soft palate for velopharyngeal closure can be assessed by positioning the endoscope in the posterior nasal cavity and asking the patient to repeat syllables beginning with fricative or plosive consonants, such as "kitty cat", and watching for bubbles while the patient holds an /s/. The tongue base, pharynx, and hypopharynx are then examined, looking for asymmetry, abnormal spontaneous movements, and pooling of secretions in the piriform sinuses and vallecula. The pharyngeal squeeze maneuver is performed by asking the patient to phonate a high-pitched, strained /i/ sound, resulting in dramatic contraction of the pharyngeal constrictors. This maneuver will demonstrate pharyngeal weakness and asymmetry, helpful both for diagnosis of a proximal neurologic lesion and for prediction of swallowing function [26, 27•].

\section{Videostroboscopy and High-Speed Imaging}

Videostroboscopy has been well established as part of the evaluation of dysphonic patients. Although it is routinely 
used during laryngoscopic examination of patients with UVFI, it is limited by difficulties capturing reliable stroboscopic signals for severely dysphonic patients and it is unclear whether the information it provides leads to changes in management [28]. In cases of vocal fold motion impairment in which flexible laryngoscopic examination is unclear, stroboscopy may provide additional information by revealing phase asymmetry and enabling detailed assessment of glottic closure. Stroboscopic findings have also been shown to correlate well with objective voice analysis and are useful for assessing post-procedure outcomes [29]. A recent study found significant differences in dynamic glottal gap area on stroboscopy between patients with RLN and SLN paralysis compared with RLN paralysis alone, suggesting that stroboscopy may be useful for determining cricothyroid muscle function as it affects vibratory control [25].

High speed laryngeal imaging has evolved as a method of laryngeal visualization that overcomes many of the limitations of videostroboscopy [30-32]. It has been proposed as a useful diagnostic tool for vocal fold paresis [33] and a method for automatic diagnosis of vocal fold paresis has been developed by use of image-analysis technology based on high-speed imaging [34]. Although high-speed imaging offers many exciting possibilities in the evaluation of dysphonic patients, currently the expense of the equipment prevents widespread use in everyday practice, and its clinical utility in vocal fold motion impairment remains to be seen. Certainly, in most cases of UVFI, standard flexible laryngoscopy is adequate for diagnosis and treatment planning. Techniques such as videostroboscopy and highspeed imaging may be helpful for evaluation of more subtle cases of vocal fold paresis and for monitoring the vibratory function of the vocal folds before and after treatment [35].

\section{Further Investigations}

\section{Imaging}

When the history and the physical examination findings do not clearly identify the cause of UVFI, further investigation of the etiology is indicated. Imaging from the origin of the vagus nerve in the skull base through the course of the RLN in the mediastinum is well established as part of the evaluation of patients with vocal fold paralysis of unknown etiology. The most commonly used imaging modalities for this purpose are chest radiography (CXR) and neck and chest computed tomography (CT) scans; magnetic resonance imaging (MRI) and ultrasound are used less frequently. There is no consensus among otolaryngologists regarding which modality is best. A recent survey of American Broncho-Esophagological Association members asked which studies are often or always necessary; CXR was believed to necessary by $69 \%$ of respondents, CT by $72 \%$, and MRI by $39 \%$ [18].

Critical evaluation of the literature reveals that the evidence is insufficient to enable a blanket recommendation for all cases, but cross sectional imaging is likely to be indicated [36]. Although some important causes of paralysis, for example intrathoracic malignancy or aortic arch aneurysm, may be detected on CXR [37], plain radiographs may miss significant thoracic pathology in $42-72 \%$ of cases of vocal fold paralysis, particularly within the left aortopulmonary window [38, 39॰]. It has been proposed that CXR should be obtained as a first step in evaluation: if negative, CT scan is obtained to rule out missed pathology; if positive, directed evaluation with further imaging studies are obtained as indicated [37]. However, cross-sectional imaging with CT or MRI is likely to be recommended for evaluation of most cases of thoracic pathology causing vocal fold immobility, and it may, therefore, be most efficient to proceed directly to cross-sectional imaging initially.

The patient's clinical history and physical examination may help to guide imaging decisions. If a high vagal lesion is suspected, an MRI may be the most useful imaging modality for evaluation of the skull base [40]. True positive findings on CT or MRI will be more likely for patients who fall into a high-suspicion group on the basis of clinical history; the number of false positive findings will be greater for those in a low-suspicion group [41]. The individual patient and treating physician must weigh the benefits of discovering potential pathology against the risks of radiation exposure, cost, and morbidity of further testing. In summary, although evidence is limited to retrospective studies, cross sectional imaging along the course of the vagus nerves and RLNs with CT or MRI is generally recommended for evaluation of UVFI without a clear etiology.

\section{Laboratory Studies}

Laboratory testing has been described as part of the evaluation of patients with UVFI without clear etiology. Such tests include rheumatoid factor, Lyme titer, erythrocyte sedimentation rate, antinuclear antibody (ANA), angiotensin-converting enzyme (ACE), syphilis testing, and blood chemistry including glucose [18]. Although results from these studies may be abnormal for medical diseases associated with UVFI, no studies support their routine use in the absence of a high clinical suspicion of a particular disease [2]. Indeed, $80 \%$ of surveyed members of the American Broncho-Esophagological Society indicated that these tests are only occasionally or rarely appropriate in cases of idiopathic UVFI [18]. 
Laryngeal Electromyography

Laryngeal electromyography (LEMG) is another technique used by some otolaryngologists to differentiate between mechanical and neurogenic causes of UVFI and to predict prognosis for recovery of vocal fold paralysis. The ability to predict poor recovery may affect the decision to proceed with early definitive surgical intervention. A survey of members of the American Broncho-Esophagological Association, found that $75 \%$ of respondents felt LEMG was at least occasionally necessary for evaluation of UVFI, although the indications, timing, and interpretation vary among clinicians [42].

In cases in which it is unclear whether immobility is caused by neurologic injury or mechanical fixation, a normal LEMG pattern strongly suggests mechanical etiology. However, cases of UVFI in which both mechanical and neurogenic etiology contribute, for example prolonged intubation, may result in diagnostic confusion [43].
Results from use of LEMG as a prognostic measure of recovery from vocal fold paralysis have varied among investigators, probably because of different techniques and interpretation among clinicians and the complex, variable, and uncertain nature of spontaneous laryngeal re-innervation. It is the unpredictability of laryngeal re-innervation that drives the desire for a reliable prognostic tool for vocal fold paralysis and has led to several studies evaluating the utility of LEMG in this regard. A meta-analysis including 503 patients that evaluated the utility of LEMG for accurate prediction of prognosis of motion recovery found that LEMG was able to predict poor prognosis with $91 \%$ accuracy, but was only able to predict good recovery $56 \%$ of the time [44*0]. Similarly, a multidisciplinary panel convened by the Neurolaryngology Study Group of the American Academy of Otolaryngology-Head and Neck Surgery found that LEMG is most useful for predicting poor functional outcome and is clinically useful as a qualitative, rather than quantitative, measure. They
Fig. 2 Examples of LEMG findings from the thyroarytenoid muscle in vocal fold paralysis. a Evidence of re-innervation with polyphasic potentials, increased amplitude, and the "picket fence" pattern of reduced recruitment.

b Polyphasic potentials. c Evidence of synkinesis with inappropriate activation of the thyroarytenoid muscle when the patient is asked to sniff (arrows)

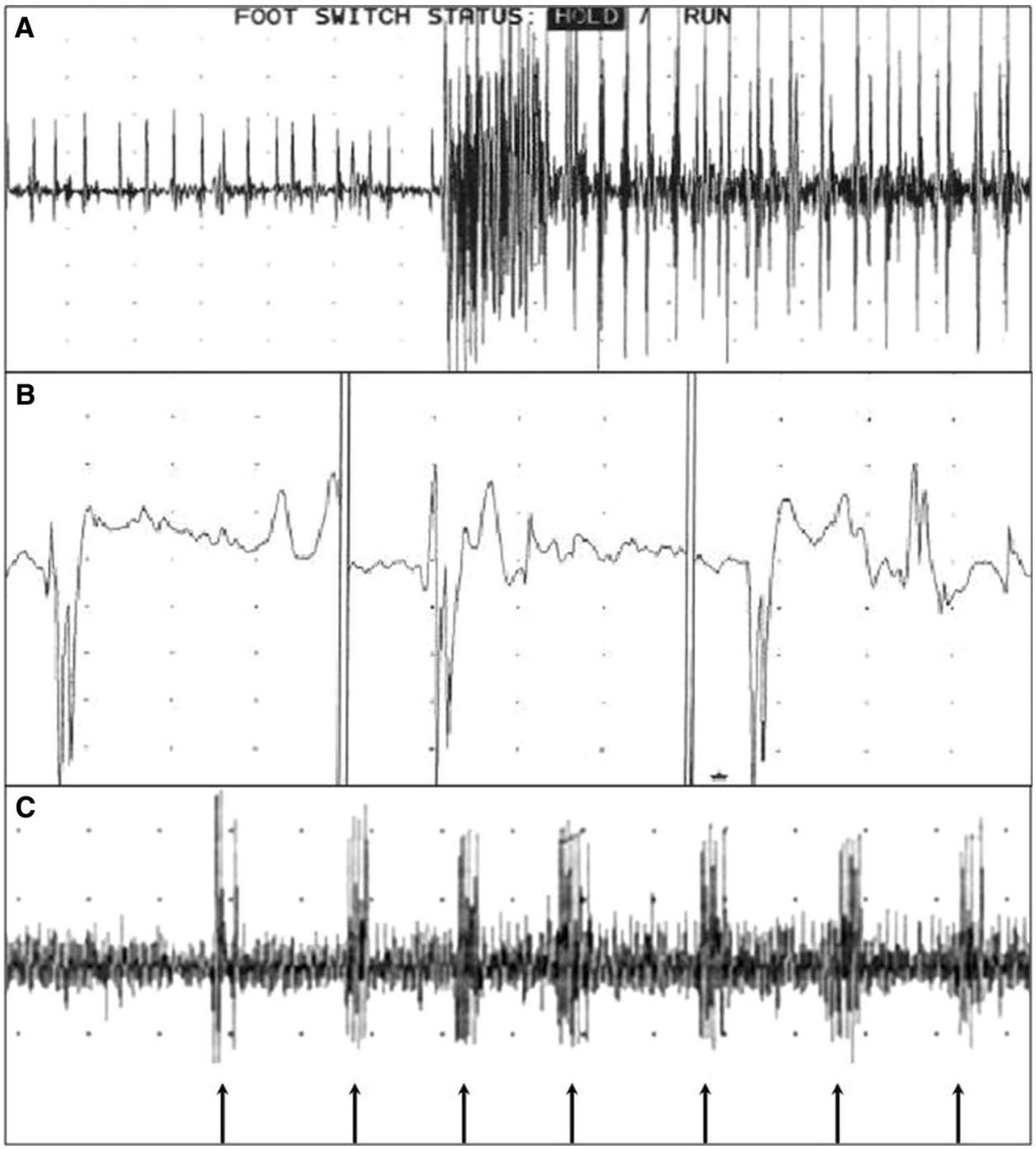


concluded that further studies are needed to standardize the practice of LEMG and determine its optimum utility and clinical value [45].

Timing of LEMG should be at least 14 days after onset of immobility, to enable Wallerian degeneration to occur and spontaneous activity to emerge, and is most useful within six months of injury. LEMG for evaluation of UVFI is most often performed using monopolar needle electrodes in the thyroarytenoid, cricothyroid, and posterior cricoarytenoid muscles, with some clinicians including the lateral cricoarytenoid, interarytenoid, and cricopharyngeus muscles [42]. Many otolaryngologists $(56 \%)$ perform diagnostic LEMG with an electromyographer for additional assistance with interpretation. Poor prognostic signs include the presence of spontaneous activity, aberrant motor unit action potential (MUAP) morphology, reduced recruitment, synkinesis, and electrical silence (Fig. 2).

Synkinesis is frequently encountered in cases of neurogenic UVFI; it results from aberrant cross-re-innervation between adductor and abductor muscle groups, and has been found to be associated with poor prognosis for functional recovery (Fig. 2c) [46]. This can be tested by having the patient perform an abductor task (sniffing) while measuring activity from an adductor muscle, and vice versa. Increased electrical activity during these tasks indicates that cross-reinnervation has occurred. A range of re-innervation exists from fully appropriate to synkinesis without any functional movement, leading to variable functional outcomes for patients with evidence of synkinesis on LEMG, depending upon the final predominant pattern of re-innervation.

\section{Formal Evaluation of Swallowing}

For patients with UVFI, the presence of aspiration and significant dysphagia may have the biggest effect on the decision to proceed with early intervention. The prevalence of aspiration among patients with UVFI is $18-38 \%$ [47-49]. Therefore, if a patient complains of dysphagia, or aspiration is suspected, further evaluation of their swallowing function should be performed. Modified barium swallow (MBS) is the recommended method for evaluation of swallowing complaints, although flexible endoscopic evaluation of swallowing (FEES) and FEES with sensory testing (FEESST) have emerged as techniques that enable safe and convenient evaluation of swallowing that can be performed in the office [27•].

\section{Conclusions}

UFVI is a highly variable clinical entity that warrants thorough evaluation to assess the functional status of the larynx and to determine the etiology. Individuals with poor cough or significant dysphagia may warrant urgent rehabilitation of glottal competence. If the cause of vocal fold immobility is not discovered despite careful history, physical examination, and laryngoscopic examination, imaging along the course of the vagus and RLNs is recommended. Routine laboratory studies are generally not indicated in the absence of clinical suspicion of a specific disease. LEMG may be useful in specific cases of vocal fold immobility to determine the neurologic status of the larynx and for prediction of poor prognosis.

\section{Compliance with Ethics Guidelines}

Conflict of Interest Robbi A. Kupfer and Tanya K. Meyer declare no conflicts of interest.

Human and Animal Rights and Informed Consent This article does not contain any studies with human or animal subjects performed by the authors.

\section{References}

Recently published papers of particular interest have been highlighted as:

- Of importance

•- Of major importance

1. Maisel RH, Ogura JH. Evaluation of vocal cord paralysis. Laryngoscope. 1974;84(2):302-16.

2. Terris DJ, Arnstein D, Nguyen HH. Contemporary evaluation of unilateral vocal cord paralysis. Otolaryngol Head Neck Surg. 1992;107(1):84-90.

3. Benninger MS, Gillen JB, Altman JS. Changing etiology of vocal fold immobility. Laryngoscope. 1998;108(9):1346-50.

4. Merati AL, Shemirani N, Smith TL, Toohill RJ. Changing trends in the nature of vocal fold motion impairment. Am J Otolaryngol. 2006;27(2):106-8.

5. Rosenthal LHS, Benninger MS, Deeb RH. Vocal fold immobility: a longitudinal analysis of etiology over 20 years. Laryngoscope. 2007;117:1864-70.

6. Rubin AD, Sataloff RT. Vocal fold paresis and paralysis. Otolaryngol Clin N Am. 2007;40:1109-31.

7. Kikura M, Suzuki K, Itagaki T, Takada T, Sato S. Age and comorbidity as risk factors for vocal cord paralysis associated with tracheal intubation. Br J Anaesth. 2007;98(4):524-30.

8. Sariego J. Vocal fold hypomobility secondary to elective endotracheal intubation: a general surgeon's perspective. J Voice. 2010;24(1):110-2.

9. Erman AB, Kejner AE, Hogikyan ND, Feldman EL. Disorders of cranial nerves IX and X. Semin Neurol. 2009;29(1):85-92.

10. Sulica L, Blitzer A, Lovelace RE, Kaufmann P. Vocal fold paresis of Charcot-Marie-Tooth disease. Ann Otol Rhinol Laryngol. 2001;110(11):1072-6.

11. Benson B, Sulica L, Guss J, Blitzer A. Laryngeal neuropathy of Charcot-Marie-Tooth disease: further observations and novel mutations associated with vocal fold paresis. Laryngoscope. 2010;120:291-6.

12. Rubin AD, Hawkshaw MJ, Moyer CA, Dean CM, Sataloff RT. Arytenoid cartilage dislocation: a 20-year experience. J Voice. 2005;19(4):687-701. 
13. Friedman AD, Kobler JB, Landau-Zemer T, Barbu AM, Burns JA. High-force simulated intubation fails to dislocate cricoarytenoid joint in ex vivo human larynges. Ann Otol Rhinol Laryngol. 2012;121(11):746-53.

14. Schwarz K, Cielo CA, Steffen N, Jotz GP, Becker J. Voice and vocal fold position in men with unilateral vocal fold paralysis. Braz J Otorhinolaryngol. 2011;77(6):761-7.

15. Hogikyan ND, Sethuraman G. Validation of an instrument to measure voice-related quality of life (V-RQOL). J Voice. 1999;13(4):557-69.

16. Jacobson BH, Johnson A, Grywalski C, Silbergleit A, Jacobson G, Benninger MS. The voice handicap index (VHI): development and validation. Am J Speech Lang Pathol. 1997;6:66-70.

17. Lundy DS, Casiano RR. "Compensatory falsetto": effects on vocal quality. J Voice. 1995;9(4):439-42.

18. Merati AL, Halum SL, Smith TL. Diagnostic testing for vocal fold paralysis: survey of practice and evidence-based medicine review. Laryngoscope. 2006;116:1539-52.

19. Hillel AD, Miller R. Bulbar amyotrophic lateral sclerosis: patterns of progression and clinical management. Head Neck. 1989;11(1):51-9.

20. Rosow DE, Sulica L. Laryngoscopy of vocal fold paralysis: evaluation of consistency of clinical findings. Laryngoscope. 2010;120:1376-82.

21. Belafsky PC, Postma GN, Reulbach TR, Holland BW, Koufman JA. Muscle tension dysphonia as a sign of underlying glottal insufficiency. Otolaryngol Head Neck Surg. 2002;127(5):448-51.

22. Jackson C, Jackson CL. The Larynx and its diseases. Philadelphia: WB Saunders; 1937.

23. Woodson GE. Configuration of the glottis in laryngeal paralysis. I: clinical study. Laryngoscope. 1993;103:1227-34.

24. Koufman JA, Walker FO, Joharji GM. The cricothyroid muscle does not influence vocal fold position in laryngeal paralysis. Laryngoscope. 1995;105:368-72.

25. Pei YC, Fang TJ, Li HY, Wong AMK. Cricothyroid muscle dysfunction impairs vocal fold vibration in unilateral vocal fold paralysis. Laryngoscope. 2014;124(1):201-6. doi:10.1002/lary. 24229.

26. Fuller SC, Leonard R, Aminpour S, Belafsky PC. Validation of the pharyngeal squeeze maneuver. Otolaryngol Head Neck Surg. 2009;140:391-4.

27. - Merati AL. In-office evaluation of swallowing: FEES, pharyngeal squeeze maneuver, and FEESST. Otolaryngol Clin N Am. 2013;46:31-39. This article reviews the techniques of in-office swallowing evaluation in detail and presents evidence for performing these techniques.

28. Harries ML. Unilateral vocal fold paralysis: a review of the current methods of surgical rehabilitation. J Laryngol Otol. 1996;110:111-6.

29. Rihkanen H, Reijonen P, Lehikoinen-Söderlund S, Lauri ER. Videostroboscopic assessment of unilateral vocal fold paralysis after augmentation with autologous fascia. Eur Arch Otorhinolaryngol. 2004;261(4):177-83.

30. Patel R, Dailey S, Bless D. Comparison of high-speed digital imaging with stroboscopy for laryngeal imaging of glottal disorders. Ann Otol Rhinol Laryngol. 2008;117(6):413-24.

31. Kendall KA. High-speed digital imaging of the larynx: recent advances. Curr Opin Otolaryngol Head Neck Surg. 2012; 20(6):466-71.

32. Woo P. Objective measures of laryngeal imaging: what have we learned since Dr. Paul Moore. J Voice. 2014;8(1):69-81. doi:10. 1016/j.jvoice.2013.02.001.

33. Mortensen M, Woo P. High-speed imaging used to detect vocal fold paresis: a case report. Ann Otol Rhinol Laryngol. 2008;117(9):684-7.
34. Voigt D, Döllinger M, Yang A, Eysholdt U, Lohscheller J. Automatic diagnosis of vocal fold paresis by employing phonovibrogram features and machine learning methods. Comput Methods Program Biomed. 2010;99(3):275-88.

35. Kimura M, Imagawa H, Nito T, Sakakibara K, Chan RW, Tayama N. Arytenoid adduction for correcting vocal fold asymmetry: high-speed imaging. Ann Otol Rhinol Laryngol. 2010; 119(7):439-46.

36. Misono S, Merati AL. Evaluation and management of unilateral vocal fold paralysis. Otolaryngol Clin N Am. 2012;45:1083-108.

37. Altman JS, Benninger MS. The evaluation of unilateral vocal fold immobility: is chest X-ray enough? J Voice. 1997;11(3):364-7.

38. Glazer HS, Aronberg DJ, Lee JK, Sagel SS. Extralaryngeal causes of vocal cord paralysis: CT evaluation. Am J Roentgenol. 1983;141(3):527-31.

39. - Song SW, Jun BC, Cho KJ, Lee S, Kim YJ, Park SH. CT evaluation of vocal cord paralysis due to thoracic diseases: a 10-year retrospective study. Yonsei Med J. 2011;52(5):831-837. This recent study retrospectively compared findings on CT and chest $X$-ray in patients with vocal fold paralysis due to thoracic disease and found that chest $X$-ray failed to identify $42 \%$ of thoracic malignancies discovered on CT scan in their series.

40. Jacobs CJ, Harnsberger HR, Lufkin RB, et al. Vagal neuropathy: evaluation with CT and MR imaging. Radiology. 1987;164:97-102.

41. Liu AY, Yousem DM, Chalian AA, Langlotz CP. Economic consequences of diagnostic imaging for vocal cord paralysis. Acad Radiol. 2001;8(2):137-48.

42. Halum SL, Patel N, Smith TL, Jaradeh S, Toohill RJ, Merati AL. Laryngeal electromyography for adult unilateral vocal fold immobility: a survey of the American Broncho-Esophagological Association. Ann Otol Rhinol Laryngol. 2005;114(6):425-8.

43. Meyer TK, Hillel AD, Blitzer A. Electromyography of Laryngeal and Pharyngeal Muscles. In: Blitzer A, Brin MF, Ranig LO, editors. Neurologic disorders of the Larynx. 2nd ed. New York: Theime; 2009.

44. • Rickert SM, Childs LF, Carey BT, Murray T, Sulica L. Laryngeal electromyography for prognosis of vocal fold palsy: a meta-analysis. Laryngoscope. 2012;122(1):158-161. This metaanalysis includes 503 patients who underwent laryngeal EMG and compares EMG findings to follow-up laryngoscopy to determine the ability of EMG to predict prognosis in cases of vocal fold paralysis.

45. Blitzer A, Crumley RL, Dailey SH, Ford CN, Floeter MK, Hillel AD, Hoffmann HT, Ludlow CL, Merati AL, Munin MC, Robinson LR, Rosen C, Saxon KG, Sulica L, Thibeault SL, Titze I, Woo P, Woodson GE. Recommmendations of the Neurolaryngology Study Group on laryngeal electromyography. Otolaryngol Head Neck Surg. 2009;140:782-93.

46. Statham MM, Rosen CA, Smith LJ, Munin MC. Electromyographic laryngeal synkinesis alters prognosis in vocal fold paralysis. Laryngoscope. 2010;120:285-90.

47. Tabaee A, Murry T, Zschommler A, Desloge RB. Flexible endoscopic evaluation of swallowing with sensory testing in patients with unilateral vocal fold immobility: incidence and pathophysiology of aspiration. Laryngoscope. 2005;115(4):565-9.

48. Bhattacharyya N, Kotz T, Shapiro J. Dysphagia and aspiration with unilateral vocal cord immobility: incidence, characterization, and response to surgical treatment. Ann Otol Rhinol Laryngol. 2002;111(8):672-9.

49. Heitmiller RF, Tseng E, Jones B. Prevalance of aspiration and laryngeal penetration in patients with unilateral vocal fold motion impairment. Dysphagia. 2000;15(4):184-7. 